\title{
Performance Analysis of Interaction-Free-Measurement-based Electron Microscopy
}

Akshay Agarwal $^{1 *}$, Vivek Goyal ${ }^{2}$, Karl K. Berggren ${ }^{1}$

${ }^{1 .}$ Department of Electrical Engineering and Computer Science, Massachusetts Institute of Technology, Cambridge, Massachusetts, USA

2. Department of Electrical and Computer Engineering, Boston University, Boston, Massachusetts, USA

${ }^{*}$ Corresponding author: akshayag@mit.edu

Interaction-free measurement (IFM) has been proposed as a method of reduced-damage electron microscopy [1-3]. Recently, with the implementation of Mach-Zehnder interferometers in conventional transmission electron microscopes (TEMs), it has become possible to potentially implement IFM in these tools [4-6]. Therefore, a comparison of the theoretical performance of IFM with conventional microscopy is of interest [7].

In this work, we theoretically analyzed the performance of IFM imaging of both opaque-and-transparent and semitransparent samples, and compared it to the performance of conventional scanning transmission electron microscopy (STEM)[8]. For opaque-and-transparent samples, we compared the performances of the two schemes using two metrics $-P_{\text {err }}$, the probability of misidentifying an opaque pixel as transparent or vice-versa, and $\bar{n}_{\text {damage }}$, the mean number of electrons required to image an opaque pixel. Figure 1(a) compares $P_{\text {err }}$ for IFM with that for conventional STEM, at a constant $\bar{n}_{\text {damage }}$ of 2.5 electrons per pixel, for $q$ (the prior probability of a given pixel being opaque) between 0 and 1 . We performed this comparison for IFM and conventional STEM both with and without a detector for scattered electrons $\left(D_{S}\right)$, to account for different microscope configurations. We can see that $P_{\text {err }}$ was lower for IFM (green dashed-dotted curve) than conventional STEM (purple solid curve) for a wide range of $q$. This includes the important limit of low $q$, which is commonly encountered for high-transparency electron microscopy samples.

In figure 1(b), we compare $P_{\text {err }}$ vs $\bar{n}_{\text {damage }}$ for IFM and STEM, for $q=0.5$. In these calculations, we included a sample re-illumination scheme based on updating a prior for each pixel of the sample after each round of illumination with a Poisson-limited electron beam, based on the statistics at the imaging detectors. The re-illumination for a pixel ceases once a stopping criterion is met. This scheme reduces $\bar{n}_{\text {damage }}$ for both IFM and STEM imaging to their ideal values - $2 / 3$ for IFM imaging with $D_{S}$ (green solid curve with square markers) and 1 for STEM imaging with $D_{s}$ (purple solid curve with circle markers). Therefore, conditional re-illumination allowed us to circumvent the Poisson statistics of the beam.

For semi-transparent samples, we treated the transparency $\alpha \in[0,1]$ as a continuous random variable. The statistics at the imaging detectors can be used to form an estimate of $\alpha$, and the performance of the estimator can be analyzed by looking at its mean squared error (MSE). For unbiased estimators, the inverse of the classical Fisher Information (FI) forms a lower bound for this MSE (Cramér-Rao bound). We found that the FI for IFM and STEM imaging was identical, shown by the solid blue curve in figure 2. Figure 2 also shows the MSE for two estimators for $\alpha-\widehat{\alpha_{1}}$ and $\widehat{\alpha_{2}}$, calculated using Monte-Carlo simulations. These estimators use the counts from the imaging detectors in different ways - $\widehat{\alpha_{1}}$ (purple dashed curve) averages over these counts to estimate $\alpha$, while $\widehat{\alpha_{2}}$ (orange dashed-dotted curve) uses the square of the difference between the counts. This analysis is important for establishing the best estimator for the pixel transparency. Future work will focus on combining this analysis with conditional re-illumination, to obtain the best possible performance for IFM imaging of semi-transparent samples[9]. 
References:

[1] A. C. Elitzur and L. Vaidman, Found. Phys. 23 (1993) p.987.

[2] W. P. Putnam and M. F. Yanik, Phys. Rev. A 80 (2009), 040902(R).

[3] P. Kruit et al., Ultramicroscopy 164 (2016), p.31.

[4] A. H. Tavabi et al., Eur. Phys. J. Appl. Phys. 78 (2017), 10701.

[5] A. Agarwal et al., Sci. Rep. 7 (2017), 1677.

[6] F. S. Yasin et al., J. Phys. D 51 (2018), 205104.

[7] S. Thomas et al., Phys. Rev. A 90 (2014), 053840.

[8] A. Agarwal, K. Berggren and V. Goyal, arXiv:1901:09702 [physics.ins-det] (2019)

[9] The authors acknowledge funding from the Gordon and Betty Moore Foundation, and the U.S. NSF under grands 1422034 and 1815896, and useful discussions with the QEM-2 collaboration.

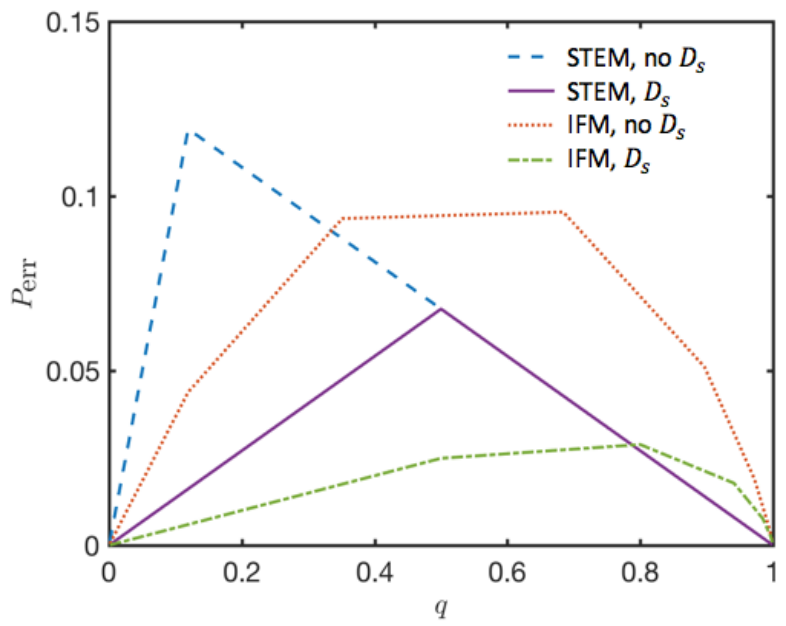

(a)



(b)

Figure 1: $P_{\text {err }}$ and $\bar{n}_{\text {damage }}$ for IFM and STEM with and without $D_{s}$. (a) $P_{\text {err }}$ vs $q$ for $\bar{n}_{\text {damage }}=2.5$ electrons per pixel. IFM (green dashed-dotted curve) outperforms STEM (purple solid curve) for $q<0.8$. (b) $P_{\text {err }}$ vs $\bar{n}_{\text {damage }}$ for $q=0.5$, with conditional sample re-illumination. $\bar{n}_{\text {damage }}$ is limited to 1 for STEM and $2 / 3$ for IFM with $D_{s}$ for arbitrarily low $P_{\text {err }}$.

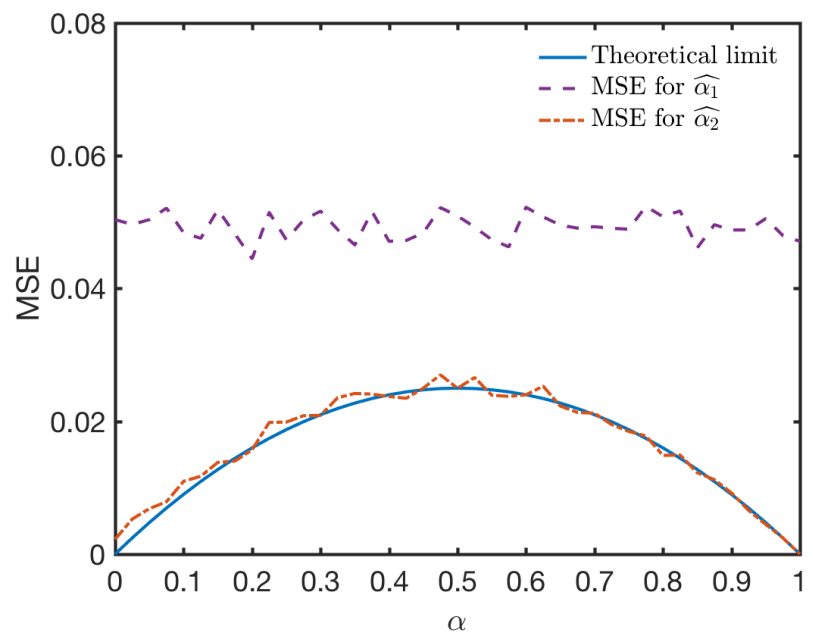

Figure 2: MSE vs $\alpha$ for IFM. The theoretical CramérRao bound is indicated by the solid blue curve. The dashed purple curve is the MSE for estimator $\widehat{\alpha_{1}}$, while the dashed-dotted ornage curve is the MSE for estimator $\widehat{\alpha_{2}}$. These MSEs were calcluated using Monte-Carlo simulations. The MSE for $\widehat{\alpha_{1}}$ is very close to the Cramér-Rao bound, and much lower than the MSE for $\widehat{\alpha_{2}}$. However, MSE for $\widehat{\alpha_{2}}$ is approximately constant for all $\alpha$. 